\title{
Response to "A Rigorous Evaluation of Methoxyflurane is Needed: Comment on 'Methoxyflurane Versus Standard of Care for Acute Trauma-Related Pain in the Emergency Setting: Protocol for a Randomised, Controlled Study in Italy (MEDITA)'"
}

\begin{abstract}
Andrea Fabbri · Giuseppe Carpinteri · Germana Ruggiano · Elisabetta Bonafede · Antonella Sblendido •
\end{abstract}
Alberto Farina $\cdot$ Amedeo Soldi

Received: February 26, 2019 / Published online: March 30, 2019

(C) The Author(s) 2019

Keywords: Acute pain; Analgesic; Emergency department; Inhaled analgesic; Injury; Methoxyflurane; Penthrox; Pre-hospital; Trauma

We welcome the critical review by Montassier and Freund on our article on the rationale and methods of the "MEDITA" study [1]. Below, we provide point-by-point answers to all comments.

Methoxyflurane is currently being introduced in Europe, and its use is supported by

Enhanced Digital Features To view enhanced digital features for this article go to https://doi.org/10.6084/ m9.figshare.7808861.

\section{A. Fabbri}

Department of Emergency Medicine, Morgagni-

Pierantoni Hospital Forlì, Forlì, Italy

G. Carpinteri

Department of Emergency Medicine, Vittorio

Emanuele University Hospital, Catania, Italy

\section{G. Ruggiano}

Emergency Medicine Department, Santa Maria

Annunziata Hospital, Florence, Italy

E. Bonafede

Bioikòs Ambiente S.r.l., CRO YGHEA Division, Bologna, Italy

\section{A. Sblendido · A. Farina $(\bowtie) \cdot$ A. Soldi}

Medical Affairs Department, Mundipharma

Pharmaceuticals srl, Milan, Italy

e-mail: alberto.farina@mundipharma.it robust data in the literature (including the STOP! study [2]) and by extensive experience in clinical practice, particularly in Australia. Although such data are adequate to support the introduction into clinical practice in Europe, further head-to-head studies vs. the best currently used therapeutic options, in terms of effectiveness and rapidity of onset, are needed. These data will help to identify methoxyflurane's place in therapy, which today is a promising option with potential advantages compared with the drugs on the market. There are now emerging data in Europe: the "InMediate" study [3], which randomised 310 patients with trauma pain to methoxyflurane or standard of care (SoC) analgesia, has recently been completed in Spain. Preliminary results indicate that methoxyflurane significantly $(p<0.0001)$ reduced pain severity compared with SoC up to 20 min after the start of treatment [4]. Another ongoing study, "PenASAP", will randomise 360 patients with trauma pain in a double-blind fashion to Penthrox or placebo, administered in addition to SoC [5]. These studies, together with the MEDITA study, which is the subject of this letter [1], all conducted with high methodological and qualitative standards, aim to resolve the uncertainties that currently persist.

Oral analgesia was not used to treat patients with moderate pain (NRS 4-6) in the MEDITA study since intravenous (IV) therapy is the common clinical practice in Italy where the 
study was conducted, although it should be underlined that there is wide heterogeneity in the therapeutic approach and oligoanalgesia still represents a widespread issue. The use of IV formulations is consistent with Italian Intersociety Recommendations on pain management in the emergency setting [6]. Likewise, in the Spanish InMediate study, oral agents were administered to only $10.7 \%$ of patients in the SoC group, although it should be noted that the majority of patients $(76.5 \%)$ had severe pain $(\mathrm{NRS} \geq 7)$. Moreover, we think that in the context of a clinical study, to evaluate the net efficacy of the molecule, it is more appropriate to use an IV formulation to make the comparison more homogeneous and to obtain head-tohead data vs. the most effective therapeutic option. A comparison vs. oral drugs would have given an obvious result because of the latency in the onset of action. A pharmacoeconomic analysis will be conducted separately at the end of the study.

The primary pain intensity end point in the "MEDITA" study is assessed over the first $10 \mathrm{~min}$ because of the rapid action of the drug and represents the most challenging comparison for methoxyflurane. By definition, IV medicinal products are immediately ready to act. This also correlates with the indication of methoxyflurane: emergency relief of moderate-to-severe pain in conscious adult patients with trauma and associated pain and the unmet need of receiving an easy-to-use, rapid-acting and effective first-line analgesic treatment for trauma-related pain. Methoxyflurane is already known to be effective at $30 \mathrm{~min}$, so the 10-min end point in the MEDITA trial provides additional information about its efficacy profile. Long duration of action is not the goal of methoxyflurane treatment. In any case, intermittent administration produces a longer analgesic effect, and if required there is the option to administer a second 3-ml dose in clinical practice. While the Penthrox inhaler indeed requires responsible disposal [7], other analgesics also have challenges in terms of consumables that are single use and non-recyclable (e.g. syringes, flasks, cannulas, needles, etc.).

Finally, Mundipharma strongly encourages and supports independent research as far as possible, and we agree that third parties have an important role to play in future investigations of methoxyflurane.

\section{ACKNOWLEDGEMENTS}

PENTHROX $^{\circledR}$ is a registered trademark of Medical Developments International Ltd.

Funding. This work was sponsored by Mundipharma Pharmaceuticals srl. No article processing charges were received by the journal for the publication of this letter.

Authorship. All named authors meet the International Committee of Medical Journal Editors (ICMJE) criteria for authorship for this article, take responsibility for the integrity of the work as a whole and have given final approval to the version to be published.

Disclosures. Elisabetta Bonafede is an employee of the clinical research organization conducting the study. Antonella Sblendido is an employee of Mundipharma Pharmaceuticals srl. Amedeo Soldi is an employee of Mundipharma Pharmaceuticals srl. Alberto Farina is an employee of Mundipharma Pharmaceuticals srl. Andrea Fabbri, Giuseppe Carpinteri and Germana Ruggiano have nothing to disclose.

Compliance with Ethics Guidelines. This article is based on previously conducted studies and does not contain any studies with human participants or animals performed by any of the authors.

Peer Review. Please note, contrary to the journal's standard single-blind peer review process, as a letter this article underwent review by a member of the journal's Editorial Board.

Open Access. This article is distributed under the terms of the Creative Commons Attribution-NonCommercial 4.0 International License (http://creativecommons.org/licenses/ by-nc/4.0/), which permits any noncommercial use, distribution, and reproduction in any medium, provided you give appropriate credit 
to the original author(s) and the source, provide a link to the Creative Commons license, and indicate if changes were made.

\section{REFERENCES}

1. Fabbri A, Carpinteri G, Ruggiano G, Bonafede E, Sblendido A, Farina A, Soldi A, MEDITA Study Group. Methoxyflurane versus standard of care for acute trauma-related pain in the emergency setting: protocol for a randomised, controlled study in Italy (MEDITA). Adv Ther. 2019;36(1):244-56.

2. Coffey F, Wright J, Hartshorn S, Hunt P, Locker T, Mirza K, Dissmann P. STOP!: a randomised, doubleblind, placebo-controlled study of the efficacy and safety of methoxyflurane for the treatment of acute pain. Emerg Med J. 2014;31(8):613-8.

3. Borobia Pe'rez AM, Capilla Pueyo R, Casal Codesido JR, InMEDIATE Group, et al. Phase IIIb, open label randomised clinical trial to compare pain relief between methoxyflurane and standard of care for treating patients with trauma pain in Spanish
Emergency Units (InMEDIATE): study protocol. IBJ Clin Pharmacol. 2017;1:e0008.

4. Borobia Pérez A, García Collado S, Carballo Cardona C, Capilla Pueyo R, Fernández Alonso C, Pérez Torres I, et al. InMEDIATE: results of a phase IIIB, open label randomised clinical trial to compare pain relief between methoxyflurane and standard of care analgesia for treating patients with trauma pain in Spanish emergency departments. Basic Clin Pharmacol Toxicol. 2018;123(Suppl. 4):16.

5. Efficacy and Safety of Penthrox ${ }^{\circledR}$ Combined With a Standard Analgesia (SoC) in Adult Patients Admitted to the Emergency Department With Moderate to Severe Pain Associated With Trauma (Pen ASAP). NCT03798899. https://clinicaltrials.gov/ct2/show/ NCT03798899? term=methoxyflurane\&rank=8. Accessed 26 Feb 2019.

6. Raccomandazioni Intersocietarie Italiane (SIAARTI, SIMEU, SIS 118, AISD, SIARED, SICUT, IRC) sulla gestione del dolore in emergenza. 2014. http://www. aisd.it/e107_files/downloads/raccintersocietarie_it_ complete31052014.pdf Accessed 26 Feb 2019.

7. Penthrox ${ }^{\circledR}$ summary of product characteristics. 2016. https://www.medicines.org.uk/emc/medicine/31391. Accessed 26 Feb 2019. 